\title{
Proximity to people with COVID-19 and anxiety among community residents during the epidemic in Guangzhou, China
}

Jinghua Su*, Xiyuan Chen*, Ning Yang, Meng Sun and Liang Zhou

\section{Summary}

The recent outbreak of a novel coronavirus pneumonia (COVID-19) may have acute psychological consequences, both in relation to the impact of the virus itself and the restrictions imposed to tackle its spread. We conducted an online survey of 403 residents in Guangzhou, China. We found the prevalence of anxiety (defined as Generalized Anxiety Disorder-7 score $\geq 5$ ) was $37.7 \%$, and anxiety was significantly and moderately correlated with worry about COVID-19. Higher anxiety levels in community residents was associated with the presence of individuals with COVID-19 in the same building; a longer time spent each day gathering information about the virus; and choosing social media as their preferred source of information. Our findings provide an insight into the psychological support and guidance about information sources that are required in this type of public health emergency.

\section{Keywords}

Psychological impact; new coronavirus; information overload; social media; anxiety.

\section{Copyright and usage}

(c) The Author(s), 2020. Published by Cambridge University Press on behalf of the Royal college of Psychiatrists. This is an Open Access article, distributed under the terms of the Creative Commons Attribution licence (http://creativecommons.org/ licenses/by/4.0/), which permits unrestricted re-use, distribution, and reproduction in any medium, provided the original work is properly cited.

\section{Background}

At the end of 2019 a novel coronavirus pneumonia (COVID-19) outbreak emerged in Wuhan, Hubei province, China, and spread rapidly to the rest of China and beyond, resulting in worldwide major public health concern. ${ }^{1}$ To stop transmission of the virus, urgent measures were put in place by the Chinese government, ${ }^{2}$ such as the prolongation of the Spring festival vacation, strict traffic control and the demand that the public stay at home. As the number of confirmed cases and deaths increased sharply and unprecedented isolation demands were imposed on the public there was an urgent need to examine the psychological impact on the public of the outbreak and the factors associated with it.

An outbreak of contagion usually has psychological consequences among the population. The 2003 outbreak of severe acute respiratory syndrome led to social disengagement, mental stress and anxiety, which subsequently led to a higher suicide rate in Hong Kong. ${ }^{3}$ A most recent survey in China during the COVID-19 epidemic revealed that the prevalence of depression and anxiety was $50.6 \%$ and $44.7 \%$, respectively, among 1563 medical staff. ${ }^{4}$ However, little is known about the psychological impact on the public so far.

Community residents are required to follow a series of strict isolation demands, such as mandatory mask wearing, discontinuation of all forms of gathering and staying at home as far as possible. In the meantime, with the rise of the internet and smart phones, an enormous amount of information is flooding into people's lives through social media including for example WeChat, Weibo and Tik Tok. Timely reports about the epidemic can help the public to avoid areas where there are more people with the virus. However, information overload may be a risk factor for anxiety.

\section{Aims}

The aim of this survey was to explore the prevalence of anxiety among community residents in Guangzhou city, the third biggest

* Joint first authors city in China, and its association with proximity to people with COVID-19, information overload and information sources.

\section{Method}

\section{Participants and sampling}

On 7 February 2020, there were 298 confirmed COVID-19 cases in Guangzhou. Data was collected from two housing compounds (KY and HL) that had newly confirmed cases in the 5 days before the survey, and one housing compound (DF) without any confirmed or suspected cases. Individuals were eligible to participate if they lived in one of the three selected communities, were aged 18 or above and had no family members diagnosed with COVID-19. One WeChat group of residents was selected in each housing compound. We distributed a non-transmissible link to the questionnaire in these three WeChat groups and invited group members to participate in the survey. The survey was conducted between 7 and 8 February 2020.

All participants had to read the informed consent and agree to participate before they could start the survey. This study was approved by the Institutional Ethics Committee of the Affiliated Brain Hospital of Guangzhou Medical University.

\section{Instruments}

We collected data on participants' demographic characteristics, proximity to COVID-19 cases, time spent collecting information about coronavirus, favourite methods to obtain information, worry about COVID-19 and anxiety. Proximity to COVID-19 was set as high (people with COVID living in the same building), medium (people with COVID living in the same housing compound but not in the same building) and low (no people with COVID in the same housing compound).

Time spent on collecting information about coronavirus referred to daily time used in collecting information relating to COVID-19, including television, newspaper, radio, websites, social 


\begin{tabular}{|c|c|c|c|c|}
\hline & $n(\%)$ & OR & $95 \% \mathrm{Cl}$ & $P$ \\
\hline \multicolumn{5}{|l|}{ Proximity to people with COVID-19 } \\
\hline Same building & $37(9.2)$ & 2.15 & $1.06-4.36$ & 0.035 \\
\hline Same housing compound & $228(56.6)$ & 1.28 & $0.82-1.99$ & 0.282 \\
\hline No people with COVID in the housing compound & $138(34.2)$ & Reference & - & - \\
\hline \multicolumn{5}{|c|}{ Daily time spent on collecting information related to COVID-19 } \\
\hline More than $2 \mathrm{~h}$ & $96(23.8)$ & 1.84 & $1.04-3.23$ & 0.035 \\
\hline $1-2 \mathrm{~h}$ & $172(42.7)$ & 1.28 & $0.81-2.01$ & 0.281 \\
\hline$<1 \mathrm{~h}$ & $135(33.5)$ & Reference & - & - \\
\hline \multicolumn{5}{|c|}{ Choosing social media as their preferred source of information } \\
\hline Yes & $306(75.9)$ & 1.75 & $1.06-2.91$ & 0.030 \\
\hline No & $97(24.1)$ & Reference & - & - \\
\hline
\end{tabular}

media. A single item 'In general, do you worry about the current situation of COVID-19?' was used to evaluate the degree of worry. Four options were provided ranging from 'very worried' to 'not at all'.

Generalized Anxiety Disorder (GAD-7), a brief self-rated scale validated in a Chinese population and commonly used in online surveys, was used to evaluate participant' anxiety level in the past 2 weeks. ${ }^{5,6}$ There are seven items and the total score ranged from 0 to 21 , with higher scores indicating severer anxiety. The total scores on the GAD-7 were categorised into no anxiety (0-4), mild anxiety (5-9), moderate anxiety (10-14) and severe anxiety symptoms $(\geq 15)^{7}$

\section{Data analysis}

Descriptive analyses were used to describe the demographic characteristics and prevalence of anxiety. Spearman's correlation was performed to probe the correlation between worry about COVID-19 and anxiety. Ordinal logistic regression was performed to explore factors associated with participants' anxiety levels. All analyses were conducted using SPSS version 22.

\section{Results}

A total of 1420 residents from three WeChat groups were approached, and 539 participants completed the questionnaire. In total, 136 were excluded because they were aged under 18, were not from the three sample housing compounds or had family members diagnosed with COVID-19. Finally, 403 participants were included in the analyses (response rate $28.4 \%$ ). Twenty-eight participants who did not know that there were confirmed cases in their housing compound were classified into the group with no confirmed cases.

\section{Characteristics of participant}

The mean age of the 403 participants was $42($ s.d. $=11.2)$ years, $68.5 \%$ were women, $87.3 \%$ were married and $79.7 \%$ had a college education or higher.

\section{Prevalence of anxiety}

The mean score on the GAD-7 was 4.4 (s.d. $=4.8$ ). The prevalence of anxiety was $37.7 \%$ (152/403); $23.1 \%$ had mild anxiety, $9.7 \%$ had moderate anxiety and 5.0\% had severe anxiety. Significant and moderate correlation was found between worry about COVID-19 and GAD-7 scores $(r=0.545, P<0.001)$.

\section{Multivariate ordinal logistic regression}

Proximity to patients with COVID-19, time spent on collecting information about coronavirus and preferring to use social media as their information source were included in the multivariate analysis. Demographic characteristics including age, gender, marital status, education level and family income were also included. Table 1 shows that after controlling for demographic characteristics, the presence of cases of COVID-19 in the same building, spending more than $2 \mathrm{~h}$ each day collecting information about COVID-19 and preferring to use social media as their information source were significantly associated with anxiety, whereas no significant association was found between all demographic characteristics and anxiety.

\section{Discussion}

In this study, we focused on anxiety symptoms rather than on anxiety disorder because with anxiety symptoms interventions are possible at the community level. Anxiety was highly prevalent and is significantly correlated to worry about COVID-19 during the peak period of the COVID-19 outbreak. In a previous survey of 8917 community residents in Shanghai in 2014, only 7.8\% reported GAD-7 scores of 5 or higher, much lower than that in our study. ${ }^{8}$ The moderate correlation between anxiety and worry about COVID-19 indicates that the elevated anxiety among community residents is likely a consequence of the COVID-19 epidemic. Anxiety was not associated with demographic characteristics in our study, which indicates the impact of the COVID-19 epidemic may be universal in community residents.

The presence of people with COVID-19 in the same building was associated with anxiety. This may be a reasonable emotional response to actual threat. There have been several news reports of multiple cases on different floors and in different apartments in the same buildings. Although individuals with confirmed/suspected cases were admitted to hospital immediately after being identified, more social and psychological support is needed for those who live in the same building.

Spending more than $2 \mathrm{~h}$ per day collecting information related to COVID-19, particularly through social media, was associated with anxiety. Our results are similar to findings from a previous study that indicated that post-traumatic stress is associated with indirect exposure to $9 / 11$ through media. ${ }^{9}$ Social media often reports unconfirmed and/or unofficial news that may increase anxiety. Advice about an appropriate amount of time to spend collecting information through reliable sources should be given.

There are several limitations to our study. First, this is not a representative sample. Socioeconomic status is high among residents of the selected housing compounds. Hence, the prevalence of anxiety 
in our study is not generalisable. Second, we did not include other factors such as pre-existing mental disorders that may be related to anxiety. Third, the cross-sectional nature of this study prevents examination of causality. In conclusion, people who are experiencing higher levels of anxiety may spend more time collecting information about COVID-19.

Jinghua Su, Department of Social Psychiatry, The Affiliated Brain Hospital of Guangzhou Medical University (Guangzhou Huiai Hospital), China; Xiyuan Chen, Department of Social Psychiatry, The Affiliated Brain Hospital of Guangzhou Medical University

(Guangzhou Huiai Hospital), China; Ning Yang, Department of Social Psychiatry, The Affiliated Brain Hospital of Guangzhou Medical University (Guangzhou Huiai Hospital), China; Meng Sun, Department of Social Psychiatry, The Affiliated Brain Hospital of Guangzhou Medical University (Guangzhou Huiai Hospital), China; Liang Zhou (iD), Department of Social Psychiatry, The Affiliated Brain Hospital of Guangzhou Medical University (Guangzhou Huiai Hospital), China

Correspondence: Liang Zhou. Email: liangzhou_csu@vip.163.com

First received 5 Mar 2020, final revision 6 May 2020, accepted 17 Jun 2020

\section{Data availability}

All data included in this study are available upon request by contact with the corresponding author.

\section{Author contributions}

L.Z. designed the study and wrote the protocol. J.S., N.Y. and X.C. collected the data. J.S. undertook the statistical analysis and wrote the first draft of the manuscript and worked with L.Z., X.C. and M.S. on final preparation.

\section{Declaration of interest}

ICMJE forms are in the supplementary material, available online at https://doi.org/10.1192/bjo. 2020.59

\section{References}

1 Zhu N, Zhang D, Wang W, Li X, Yang B, Song J, et al. A novel coronavirus from patients with pneumonia in China, 2019. N Engl J Med 2020; 382: 8.

2 Xiang YT, Yang Y, Li W, Zhang L, Zhang Q, Cheung T, et al. Timely mental health care for the 2019 novel coronavirus outbreak is urgently needed. Lancet Psychiatry 2020; 7: 228-9.

3 Yip PS, Cheung YT, Chau PH, Law YW. The impact of epidemic outbreak: the case of severe acute respiratory syndrome (SARS) and suicide among older adults in Hong Kong. Crisis 2010; 31: 86-92.

4 Kang L, Li Y, Hu S, Chen M, Yang C, Yang BX, et al. The mental health of medical workers in Wuhan, China dealing with the 2019 novel coronavirus. Lancet Psychiatry 2020; 7: e14.

5 He YX, Li CB, Qian J, Cui HS, Wu YW. Reliability and validity of a generalized anXiety disorder scale in general hospital outpatients. Shanghai Arch Psychiatry 2010; 22: 200-3.

6 Zhou SJ, Zhang LG, Wang LL, Guo ZC, Wang JQ, Chen JC, et al. Prevalence and socio-demographic correlates of psychological health problems in Chinese adolescents during the outbreak of COVID-19. Eur Child Adolesc Psychiatry 2020; 29: 749-58.

7 Spitzer RL, Kroenke K, Williams JB, Lowe B. A brief measure for assessing generalized anxiety disorder: the GAD-7. Arch Intern Med 2006; 166: 1092-7.

8 Qi X. Emotional Problems and Service Needs of Community Residents. Shanghai Jiao Tong University, 2014.

9 Silver RC, Holman EA, McIntosh DN, Poulin M, Gil-Rivas V. Nationwide longitudinal study of psychological responses to September 11. JAMA 2002; 288: 1235-44. 